\title{
Correlation between abnormal thyroid function test and thyroid autoimmunity: a systematic review and meta-analysis
}

\author{
Ning Xiao ${ }^{1,2 \#}$, Yingying $\mathrm{Li}^{1,2 \#}$, Wangjin Chen ${ }^{1,2}$, Ying Liu ${ }^{1,2}$, Dongyu $\mathrm{Li}^{1,2}$ \\ ${ }^{1}$ Department of Health Management \& Institute of Health Management, Sichuan Provincial People's Hospital, University of Electronic Science and \\ Technology of China, Chengdu, China; ${ }^{2}$ Chinese Academy of Sciences Sichuan Translational Medicine Research Hospital, Chengdu, China \\ Contributions: (I) Conception and design: N Xiao, Y Li, Y Liu, D Li; (II) Administrative support: W Chen; (III) Provision of study materials or \\ patients: N Xiao, Y Li, Y Liu; (IV) Collection and assembly of data: All authors; (V) Data analysis and interpretation: All authors; (VI) Manuscript \\ writing: All authors; (VII) Final approval of manuscript: All authors. \\ "These authors contributed equally to this work. \\ Correspondence to: Dongyu Li; Ying Liu. Sichuan Provincial People’s Hospital, University of Electronic Science and Technology of China, No. 32, \\ West 2, Section 1 Ring Road, Qingyang District, Chengdu, China. Email: fordongyu@163.com; liuhaoran636@163.com.
}

\begin{abstract}
Background: This study was to analyze the correlation between abnormal thyroid function detection and thyroid autoimmune disease, so as to provide theoretical guidance for clinical diagnosis of thyroid autoimmune disease.

Methods: The Chinese databases were searched with a combination of words "thyroid", "thyroid autoimmune disease", "thyroid function testing", "meta-analysis". The entire database was searched in English language databases with the search terms "thyroid", "thyroid autoimmune disease", "thyroid function test ", "meta-analysis". Review manager software was applied for meta-analysis.

Results: A total of 8 articles were finally included. 4 articles reported the T3 level, showing $\mathrm{P}=0.95, \mathrm{I}^{2}=0 \%$, odds ratio $(\mathrm{OR})=2.39,95 \%$ confidence interval $(\mathrm{CI}): 0.79-7.25 ; 3$ articles reported the T4 levels, showing $\mathrm{P}=0.81, \mathrm{I}^{2}=0 \%, \mathrm{OR}=2.16,95 \% \mathrm{CI}: 0.43-10.71$; and 4 articles reported the thyroid stimulating hormone (TSH) level, showing $\mathrm{P}=0.48, \mathrm{I}^{2}=0 \%$, OR $=3.20,95 \%$ CI: $1.45-7.07$.

Discussion: After a systematic review of the literature, a significant difference was found in T3 and TSH levels between patients with autoimmune thyroid disease and those with a healthy thyroid, but the difference in T4 level was not significant.
\end{abstract}

Keywords: Thyroid; thyroid autoimmune disease; thyroid function test; meta-analysis

Submitted Oct 31, 2021. Accepted for publication Dec 16, 2021.

doi: 10.21037/apm-21-3534

View this article at: https://dx.doi.org/10.21037/apm-21-3534

\section{Introduction}

The thyroid is an endocrine organ and the gland of traditional Chinese medicine in vertebrates, which is the largest endocrine gland in the human body. Located beneath the thyroid cartilage in the mammalian neck, on both sides of the trachea (1). The human thyroid gland has a shape such as a shield thyroid, and is therefore called the thyroid gland. The thyroid gland regulates the body's calcium and phosphorus balance, promotes growth and development, increases energy metabolism, and regulates the metabolism of sugars, lipids as well as proteins, affecting human growth and development (2). The thyroid gland secretes calcitonin, regulates the body calcium phosphorus balance, and secretes thyroid hormone is able to promote growth and development. When thyroid function is abnormal, the main diseases include hyperthyroidism, hypothyroidism, thyroid inflammation, goiter, and thyroid tumors. Thyroid autoimmune diseases, which refer to diseases in which the body develops an immune response to self antigens, resulting in damage to its own tissue, are thyroid diseases that result from disturbances in the autoimmune system (3). 
Thyroid autoimmune diseases are mainly caused by abnormal self antigens, immune dysregulation, genetics and other factors. Thyroid autoimmune diseases occur more often in women and less frequently in men (4).

The symptoms of thyroid autoimmune diseases are mainly hyperthyroidism, fever, hair loss, fatigue, oral ulcers, and insomnia. Patients with abnormal thyroid function are more likely to have thyroid autoimmune diseases than normal people (5). With the development of modern medical technology, more and more people begin to pay attention to their health. Patients who were not checked out due to the relative shortage of medical conditions in the past have also been diagnosed due to the development of science and technology and economic progress. Therefore, patients who have been diagnosed have gradually increased in recent years (6). In hyperthyroidism or hypothyroidism, the levels of hormones secreted by the gland into the circulation are altered accordingly, multiple antibodies are present in the serum of patients with autoimmune thyroid diseases, and current clinical programs for thyroid function testing generally include T3, T4, TSH (thyroid-stimulating hormone), and so on (7). The thyroid can promote cell differentiation and maintain metabolic balance in the body.

$\mathrm{T} 3$ and $\mathrm{T} 4$ are iodinated tyrosine residues on Thyroidglobulin $(\mathrm{Tg})$ in thyroid follicular epithelial cells. The main daily product of the thyroid gland is $\mathrm{T} 4$, and its output is about 10-20 times that of T3, but its affinity with nuclear receptors is one-tenth that of $\mathrm{T} 3$, so $\mathrm{T} 3$ has a stronger physiological activity. Almost all T3 and T4 are combined in the blood. About three-quarters are bound to globulin, and the remaining quarter is bound to other plasma proteins. Free and non-protein-bound thyroid hormone has physiological activity. The only source of endogenous T4 depends on the secretion of the thyroid gland. The secretion of thyroid T3 accounts for one-fifth, and the remaining four-fifths are transformed by $\mathrm{T} 4$ by deiodinase (8). Therefore, T4 can be referred to as the precursor of T3. When the human body does not have enough iodine intake, the thyroid gland will give priority to the synthesis of T3. TSH is secreted by the pituitary gland, and the specific location is in the lower anterior lobe. The level of TSH and its activity change inversely with the concentration of thyroxine, which is an important indicator of hyperthyroidism and hypothyroidism (9).

In this paper, the subjects with thyroid autoimmune diseases were tested for abnormalities in their thyroid function, and a meta-analysis of the correlation between the two was performed. In this study, we selected articles published since the construction of the library about the correlation between abnormal thyroid function tests and thyroid autoimmunity, comprehensively analyzed and evaluated them, and summarized the correlation between them, so as to further explore and provide theoretical guidance for better diagnosis of thyroid autoimmune diseases in the clinic.

We present the following article in accordance with the PRISMA reporting checklist (available at https://dx.doi. org/10.21037/apm-21-3534).

\section{Methods}

\section{Literature search}

This meta-analysis used an intelligent computer to conduct a comprehensive search on the China Knowledge Network (CNKI) database, China Biomedical Literature Database, Wanfang Database, Weipu Database, Baidu Academic, CMCI, Medline, Embase, PubMed, and other databases.

The articles were retrieved from the database, which were published from the establishment of the database to August 1, 2021, to analyze the correlation between abnormal thyroid function detection and thyroid autoimmunity. The compound logic retrieval and Boolean logic retrieval were adopted to select the related documents. In the Chinese database, the words "thyroid", "thyroid autoimmune diseases", "thyroid function testing", and "meta-analysis" were used to combine each word, and then all the documents were searched. In the English database, the "Thyroid", "Thyroid autoimmune disease", "Thyroid function test", and "Meta-analysis" were undertaken as search terms to search the entire database. This study was based on the Rev Man 5.3 software provided by the Cochrane system to evaluate the quality of the literature.

For the several vocabularies listed in the previous paragraph, they were combined in multiple ways and then the articles were searched. After this step was repeated many times, the references would be finalized. A search engine was adopted to track the last included articles to obtain complete information. It should read the contents of the articles carefully, and then record the general information to obtain complete information. If there was no complete information in the article, the authors of the articles should be contacted as soon as possible to obtain the information for record. At the same time, it was necessary to get in touch with professional practitioners and industry experts in the field to obtain the latest research progress. 


\section{Literature inclusion and exclusion criteria}

Inclusion criteria were given as follows: (I) the included article was a randomized controlled study; (II) the language of the final included article was English; (III) the included research subjects were patients with thyroid autoimmune disease; (IV) the patient cooperated with the doctor's followup diagnosis and treatment, and the index of pathological analysis was within the $95 \%$ confidence interval (CI).

Exclusion criteria were as follows: I. the articles mentioning the research object with other thyroid diseases; II. articles which were duplicated or without complete data; III. case-control studies, relevant conference materials, literature reviews, individual case research reports, lectures, and review literature; IV. patients who had taken iodinecontaining drugs; and patients who had used drugs that affect the thyroid.

After the final obtained documents were read through by two experts, the topics, abstracts, and full texts were read through, and then a comprehensive screening was performed. Before screening, three preliminary experiments were required. The two experts each gave their final opinions. If the final conclusions were inconsistent, it should discuss again to obtain a consistent conclusion. If the results can't be discussed, it could seek the help of a third party to obtain consistent comments.

\section{Observation indexes}

The result indicators were whether the T3 level, T4 level, and TSH level were abnormal.

\section{Data extraction}

The final selected articles would be screened by two professionals in the industry after reading the full text of the article, and three pre-experiments would be done before screening. If the opinions of the two experts diverged, they needed to be discussed again to come up with a consistent conclusion. If it failed to get a consistent conclusion, it could seek third-party advice to give a consistency evaluation. The general data of the extracted article included: I. the name of the first author; II. the publication year of the article; III. the sample size of the literature experimental group; IV. the sample size of the literature control group; and V. whether the T3 level, T4 level, and TSH level were abnormal.

\section{Bias risk and quality assessment}

Two industry experts conducted a bias risk assessment on the final included articles, and needed to communicate and discuss or find a third party to finally obtain a consistency evaluation. This meta-analysis was a quality assessment based on the recommendations given by the National Institute of Clinical Excellence (NICE). The scores ranged from $0-8$ points, and the scores for high-quality research documents were $6-8$ points; those that were evaluated as medium-quality were scored $3-6$ points; those that were evaluated as low-quality were scored $0-2$ points. The finally obtained articles were submitted to two researchers to evaluate the quality separately. When there was a disagreement, it could discuss and get a consistent conclusion. If a consistent conclusion could not be obtained, it should promptly obtain the help of a third party to give the conclusion. When the risk bias of the article was evaluated, two professionals judged the various indicators of the included articles. There were three results, namely "high risk bias", "low risk bias", and "unclear". When the opinions were inconsistent, it could seek third-party help in time after the discussion fails, and then give a consistent result.

\section{Statistical analysis}

In this study, the statistical analysis software StataSE14.0 was used to combine the effect size. The effect size indicator of the binary variable was the odds ratio (OR); the effect size indicator of the continuous variable was the standardized mean difference (MD). Meta-analysis needed to be tested for heterogeneity. When the result of heterogeneity test was $\mathrm{P} \geq 0.1$ and $\mathrm{I}^{2} \leq 50 \%$, it indicated that the difference was not significant and homogeneity, so the fixed effects model (FEM) was used for meta-analysis. When the heterogeneity test of meta-analysis was $\mathrm{P}<0.1$ and $\mathrm{I}^{2}>50 \%$, the researched articles were not homogeneous, and the heterogeneity was significant, so the random effects model (REM) was used in the meta-analysis. In this way, the heterogeneity can be reduced as much as possible in the calculation, and the comprehensive influence on the results of the literature research can be reduced, thereby promoting the reliability of the results. When the risk bias of articles was evaluated, the Rev Man 5.3 software was used to evaluate the risk of bias, and $95 \%$ CI was used for each indicator effect. 


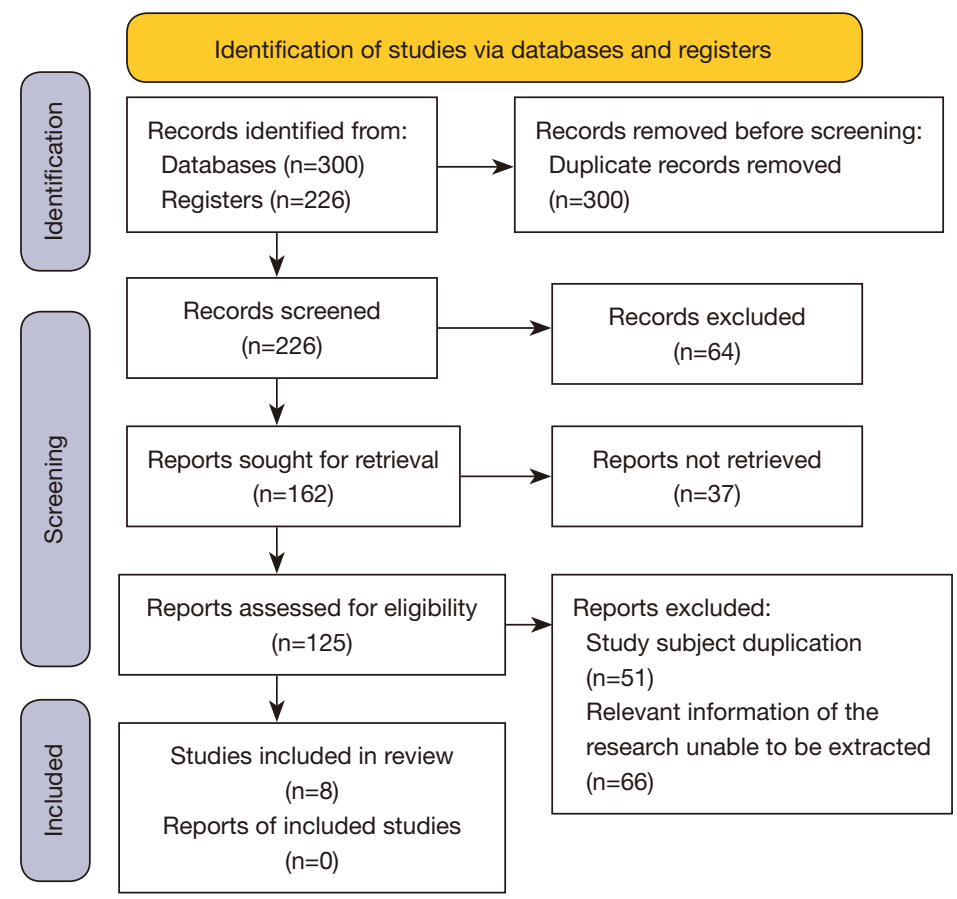

Figure 1 Flow chart of retrieval process.

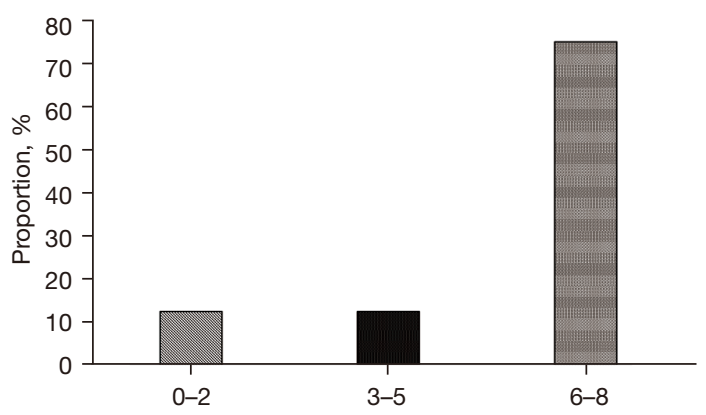

Figure 2 Results of quality assessment of included articles according to National Institute of Clinical Excellence (NICE) scoring criteria.

\section{Results}

\section{Search results and basic information of included articles}

When the articles were searched, a combination strategy was adopted. The search results showed that 300 articles were obtained from the English database and 226 articles were obtained from the Chinese database, with a total of 526 articles. After 300 duplicate articles were excluded, 226 articles remained. After the titles and abstracts of the articles were read, 101 articles were screened and 125 articles remained. After the contents of the articles were read, 117 articles were deleted, and 8 articles were finally included. Meta-analysis of these articles was performed. The entire retrieval process was shown in Figure 1 below.

Figure 2 was a diagram of the quality evaluation results based on the recommendations of the National Institute of Clinical Optimization in the United Kingdom. 6 articles scored 6-8 points, accounting for $75 \% ; 1$ article scored $3-5$ points, accounting for $12.5 \%$; and 1 article scored $0-2$ points, accounting for $12.5 \%$. After the process of screening, there were finally 8 articles remaining, which met the inclusion criteria. The total number of samples was 4,383 cases. It should record the general data of patients in 8 articles, including the name of the first author of the article, the publication year of the article, sample size, and outcome indicators. As shown in Table 1 below, it was the general information of the research objects included in the articles.

\section{Risk bias evaluation results}

Figure 3 and Figure 4 show the risk bias diagram of the ten finally included documents drawn by Review manager 5.3 software, Figure 3 shows the risk bias evaluation diagram of the finally included documents, and Figure 4 shows the corresponding multiple risk bias evaluation results of the 
Table 1 Basic information of the included articles

\begin{tabular}{|c|c|c|c|c|}
\hline First author & Year of publication & Sample size & Age (years old) & Outcome indicator \\
\hline Jonsdottir B (11) & 2017 & 2,433 & $10-15$ & (1) (3) \\
\hline Katahira M (12) & 2018 & 154 & $61-75$ & (1) \\
\hline Kosmeri C (13) & 2019 & 49 & $10-15$ & (3) \\
\hline Osorio JC (15) & 2017 & 51 & - & (3) \\
\hline Song J (16) & 2019 & 136 & - & (2) \\
\hline Sovetkina A (17) & 2020 & 126 & $40.8 \pm 1.1$ & (1) (2) \\
\hline
\end{tabular}

(1) T3; (2) T4; (3) TSH (thyroid-stimulating hormone).

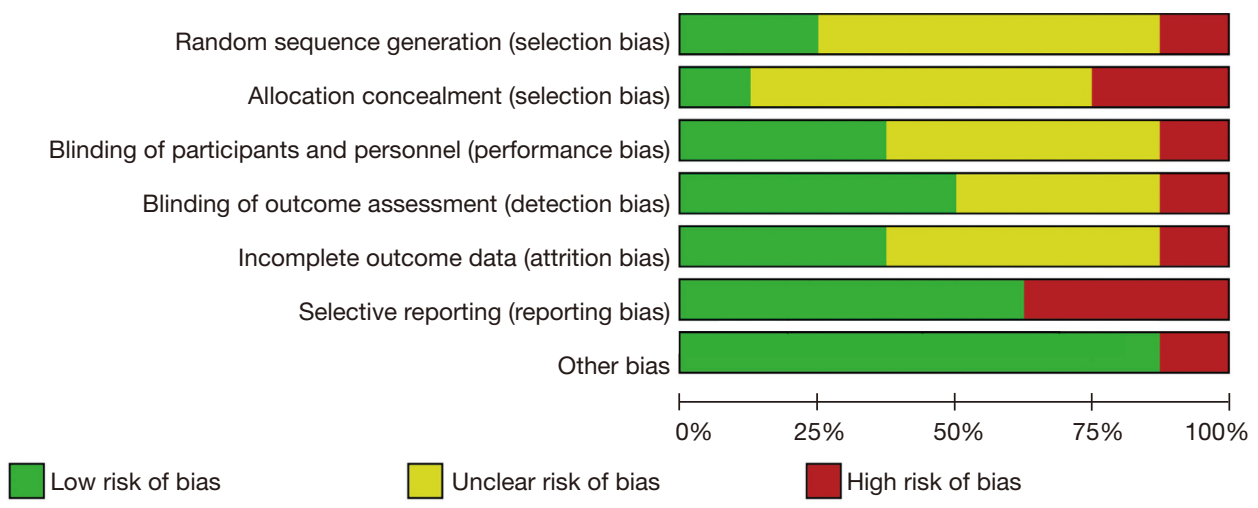

Figure 3 Risk bias assessment chart for the final 8 articles.

finally included documents.

\section{Meta-analysis of T3 level in detection of thyroid dysfunction}

Among the 8 articles that were finally included, there were 4 articles that reported the detection of T3 levels. As shown in Figure 5 below, the results of the Meta-analysis showed that the heterogeneity was not significant $(\mathrm{P}=0.95$, $\mathrm{I}^{2}=0 \%$ ), so the FEM was used. As shown in the figure, the OR value was 2.39 and $95 \%$ CI was $0.79-7.25$. The results showed that patients with thyroid autoimmune disease had abnormal T3 levels compared with patients with normal thyroid function $(\mathrm{Z}=1.54, \mathrm{P}=0.12)$. Figure 6 below was a graph of publication bias evaluation. In 4 articles, the OR value was set as the horizontal plane, the standard error was the vertical plane, and the funnel chart was drawn. As shown in Figure 6, the circles on both sides of the midline were distributed asymmetrically, indicating that the included 4 articles may have publication bias.

\section{Meta-analysis of T4 level in in detection of thyroid dysfunction}

Among the 8 articles finally included, there were 3 that reported the detection of T4 levels. As shown in Figure 7 below, the results of the Meta-analysis showed that the heterogeneity was not significant $\left(\mathrm{P}=0.81, \mathrm{I}^{2}=0 \%\right)$, so the FEM was used. The results of the analysis showed that the OR value was 2.16 and $95 \%$ CI was $0.43-10.71$, and there was no significant difference between the two groups. The T4 level of patients with thyroid autoimmune disease was basically unchanged from that of patients with normal thyroid function $(\mathrm{Z}=0.94, \mathrm{P}=0.35)$. Figure 8 below was a graph of publication bias evaluation. In 3 studies, after the T3 level of patients with thyroid autoimmune diseases was 


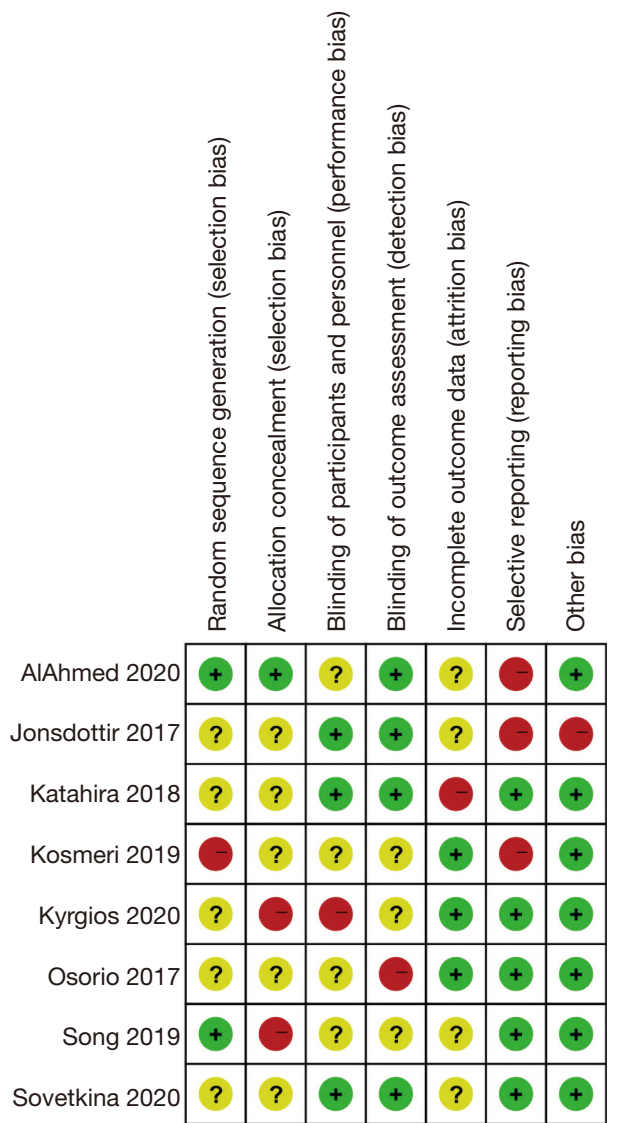

Figure 4 Results of multiple risk bias of included 10 articles.

analyzed, the OR value was set as the horizontal plane, the standard error was the vertical plane, and the funnel chart was drawn. As shown in Figure 8, the circles on both sides of the midline were distributed asymmetrically, indicating that the three included articles may have publication bias.

\section{Meta-analysis of TSH level in detection of thyroid dysfunction}

Among the 8 articles finally included, there were 4 that reported the detection of TSH levels. As shown in Figure 9 below, the results of the Meta-analysis showed that the heterogeneity was not significant $\left(\mathrm{P}=0.48, \mathrm{I}^{2}=0 \%\right)$, so the FEM was used, and the difference between the two groups was significant. The results of the analysis showed that the OR value was 3.20 and $95 \%$ CI was $1.45-7.07$, and patients with thyroid autoimmune disease had abnormal T3 levels compared with patients with normal thyroid function $(\mathrm{Z}=2.88, \mathrm{P}=0.004)$. Figure 10 below was a graph of publication bias evaluation. In 4 articles, after the TSH level of patients with thyroid autoimmune diseases was analyzed, the OR value was set as the horizontal plane, the standard error was the vertical plane, and a funnel chart was drawn. As shown in Figure 10, the distribution of circles on both sides of the midline was asymmetrical, indicating that the included 4 articles may have publication bias.

\section{Discussion}

With the development of medicine, the probability of disease being discovered also increases, which makes the treatment method more reasonable and efficient. Thyroid is more likely to be the target organ for autoimmune diseases (18). If the autoimmune system is disordered, attacking the thyroid can easily induce various thyroid diseases, such as Hashimoto's thyroiditis. The immune system disorder rate of women is higher than that of men, which may also be related to mental factors, because poor mental state can actually induce immune system disorders (19). At present, the incidence of thyroid diseases in my country is $50.96 \%$, and the prevalence of abnormal thyroid function is $15.22 \%$. Thyroid autoimmune diseases are quite different among different ethnic groups, and this difference may be related to genetic factors, environmental factors, dietary patterns, lifestyles, and economic development (20). The survey results of the correlation between iodine nutrition and the prevalence of thyroid diseases show that the prevalence of subclinical hypothyroidism will increase with the increase of iodine intake. The significant increase in the prevalence of subclinical hypothyroidism is related to the increase in the national TSH level. With the increase of iodine intake, TSH levels gradually increase, and the prevalence of hyperTSHemia also increases. Further analysis of thyroid autoantibodies found that the significant increase in the prevalence of subclinical hypothyroidism has nothing to do with thyroid autoimmunity; and only non-autoimmune subclinical hypothyroidism will increase with the increase of iodine intake. Iodine excess is only related to clinical hyperthyroidism and subclinical hypothyroidism, while iodine deficiency is related to most thyroid diseases. The risk of iodine deficiency exceeds iodine excess. Therefore, it is necessary to insist on eating iodized salt while controlling thyroid diseases. After the articles were retrieved, 8 articles were finally included. The quality of articles was evaluated, 6 articles scored $6-8$ points, accounting for $75 \% ; 1$ article scored $3-5$ points, accounting for $12.5 \%$; and 1 article scored $0-2$ points, accounting for $12.5 \%$. The total number 


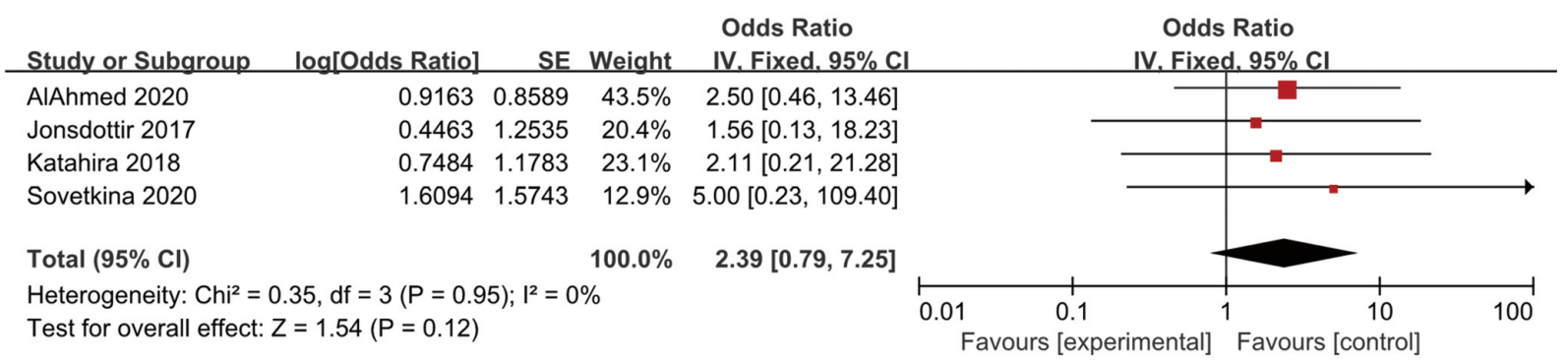

Figure 5 Forest diagram of T3 level in thyroid function tests. CI, confidence interval.

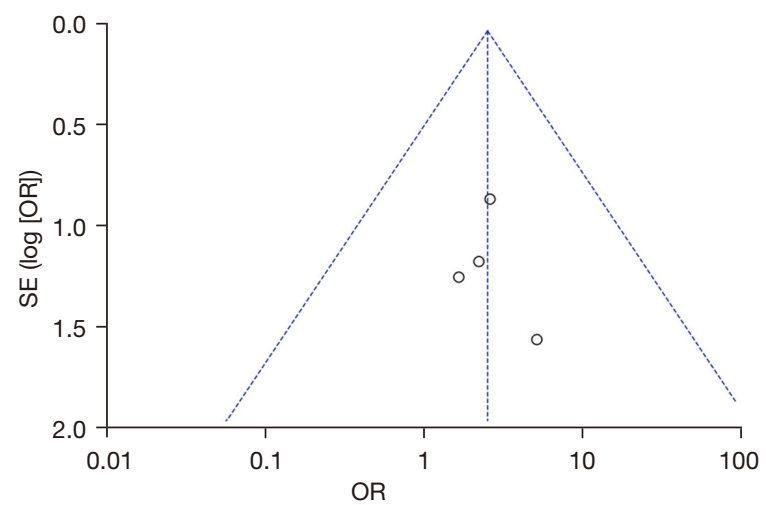

Figure 6 Funnel diagram of publication bias of T3 level in thyroid function tests. OR, odds ratio; SE, standard Error (SE).

of samples was 4383 cases. Among the 8 articles that were finally included, there were 4 articles that reported the detection of T3 levels. The results of Meta-analysis showed that its heterogeneity was not significant $\left(\mathrm{P}=0.95, \mathrm{I}^{2}=0 \%\right)$, so a FEM was used. As shown in the figure, the OR value was 2.39 and $95 \%$ CI was $0.79-7.25$, and the results showed that patients with thyroid autoimmune disease had abnormal T3 levels compared with patients with normal thyroid function $(\mathrm{Z}=1.54, \mathrm{P}=0.12)$. Among the 8 articles finally included, there were 3 articles that reported the detection of T4 levels. As shown in Figure 7, the results of the Meta-analysis showed that the heterogeneity was not significant $(\mathrm{P}=0.81$, $\mathrm{I}^{2}=0 \%$ ), so the FEM was used. The results of the analysis showed that the OR value was 2.16 and $95 \%$ CI was $0.43-$ 10.71 , and there was no significant difference between the two groups. There was basically no change in the T4 level of patients with thyroid autoimmune disease compared with patients with normal thyroid function $(\mathrm{Z}=0.94, \mathrm{P}=0.35)$. Among the 8 articles finally included, there were 4 articles that reported the detection of TSH levels. As shown in
Figure 9, the results of the Meta-analysis showed that the heterogeneity was not significant $\left(\mathrm{P}=0.48, \mathrm{I}^{2}=0 \%\right)$, so the FEM was used, and the difference between the two groups was significant. The results of the analysis showed that the OR value was 3.20 and $95 \%$ CI was $1.45-7.07$, and patients with thyroid autoimmune disease had abnormal T3 levels compared with patients with normal thyroid function $(\mathrm{Z}=2.88, \mathrm{P}=0.004)$. After the T3, T4, and TSH levels of patients with thyroid autoimmune disease were analyzed, the OR value was set as the horizontal plane, the standard error was the vertical plane, and the funnel chart was drawn. The distribution of circles on both sides of the midline was asymmetrical, indicating that the included literature may have publication bias.

\section{Conclusions}

This meta-analysis study used compound logic retrieval and Boolean logic retrieval, and a total of 8 articles were included to analyze the correlation between thyroid autoimmune diseases and abnormal thyroid function tests. The results revealed that the abnormal analysis of thyroid function detection showed that patients with thyroid autoimmune disease had greater differences in T3 levels compared with healthy thyroid patients, T4 levels were not significantly different, and TSH levels were significantly different. However, there were some limitations in this study. The reasons may include a small number of patient samples, or because the patient has other diseases that affect the test results. This influence could not be removed in the experiment, so the final conclusions would have certain deviations, and they may also be affected by environmental factors or physical differences between different individuals. In short, this meta-analysis provided a reference for the clinical diagnosis of thyroid autoimmune diseases. 


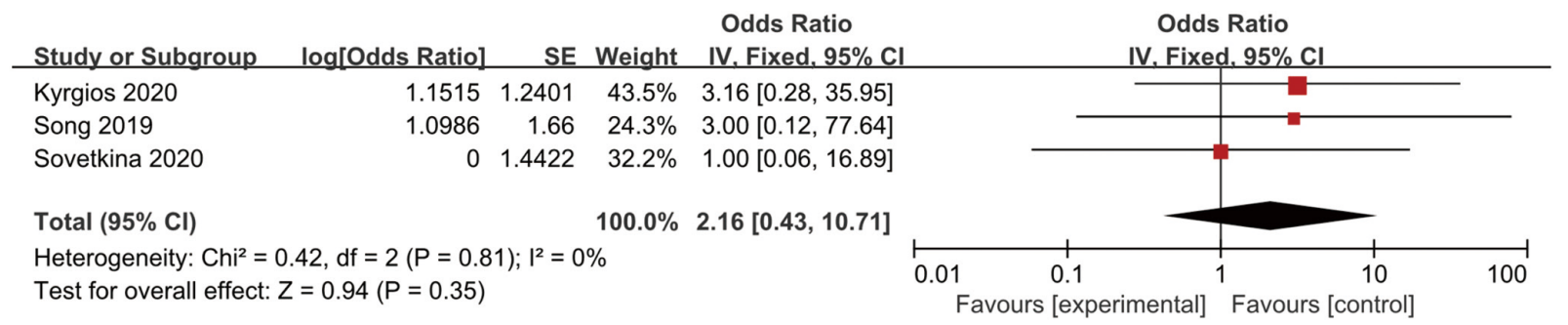

Figure 7 Forest diagram of T4 level in thyroid function tests.

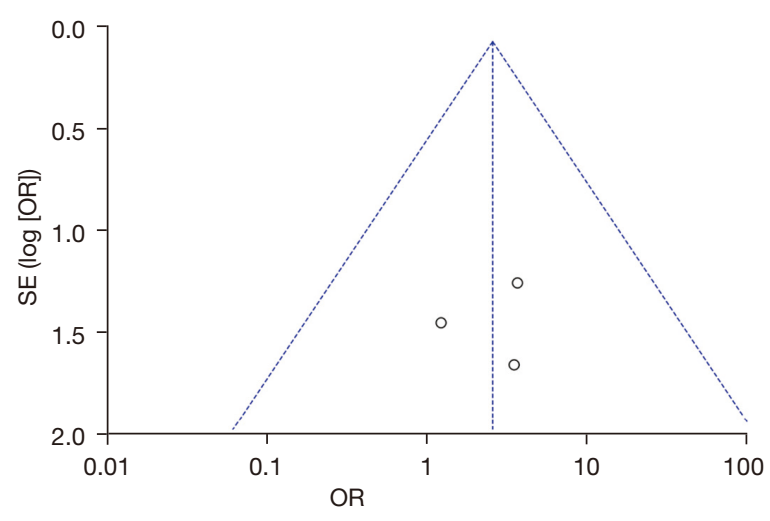

Figure 8 Funnel diagram of publication bias of T4 level in thyroid function tests.

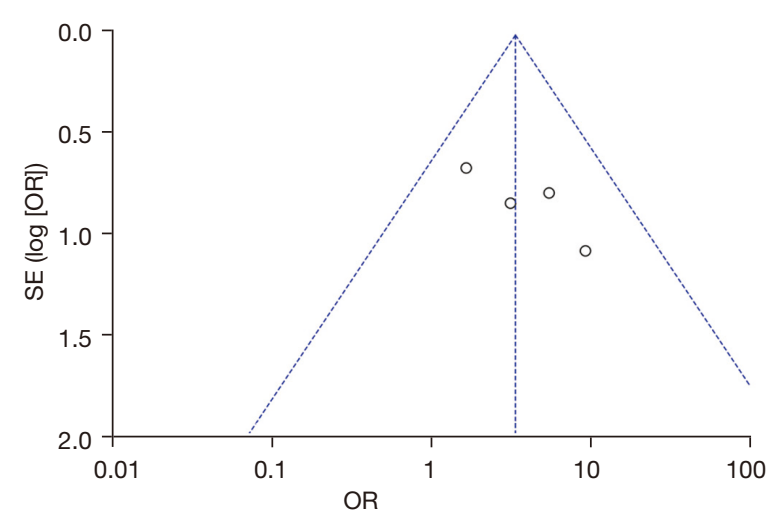

Figure 10 Funnel diagram of publication bias of thyroid stimulating hormone (TSH) level in thyroid function tests.

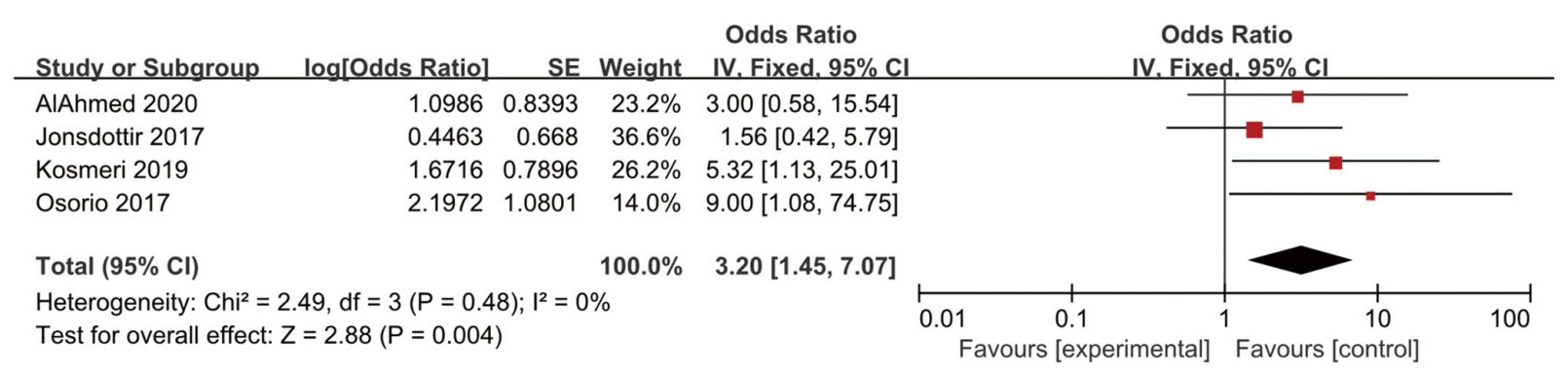

Figure 9 Forest diagram of thyroid stimulating hormone (TSH) level in thyroid function tests.

\section{Acknowledgments}

Funding: None.

\section{Footnote}

Reporting Checklist: The authors have completed the PRISMA reporting checklist. Available at https://dx.doi. org/10.21037/apm-21-3534
Conflicts of Interest: All authors have completed the ICMJE uniform disclosure form (available at https://dx.doi. org/10.21037/apm-21-3534). The authors have no conflicts of interest to declare.

Ethical Statement: The authors are accountable for all aspects of the work in ensuring that questions related to the accuracy or integrity of any part of the work are appropriately investigated and resolved. 
Open Access Statement: This is an Open Access article distributed in accordance with the Creative Commons Attribution-NonCommercial-NoDerivs 4.0 International License (CC BY-NC-ND 4.0), which permits the noncommercial replication and distribution of the article with the strict proviso that no changes or edits are made and the original work is properly cited (including links to both the formal publication through the relevant DOI and the license). See: https://creativecommons.org/licenses/by-nc-nd/4.0/.

\section{References}

1. Kim D. The Role of Vitamin D in Thyroid Diseases. Int J Mol Sci 2017;18:1949.

2. Knezevic J, Starchl C, Tmava Berisha A, et al. ThyroidGut-Axis: How Does the Microbiota Influence Thyroid Function? Nutrients 2020;12:1769.

3. Nilsson M, Fagman H. Development of the thyroid gland. Development 2017;144:2123-40.

4. Winther KH, Rayman MP, Bonnema SJ, et al. Selenium in thyroid disorders - essential knowledge for clinicians. Nat Rev Endocrinol 2020;16:165-76.

5. Alexander LF, Patel NJ, Caserta MP, et al. Thyroid Ultrasound: Diffuse and Nodular Disease. Radiol Clin North Am 2020;58:1041-57.

6. Speer G, Somogyi P. Thyroid complications of SARS and coronavirus disease 2019 (COVID-19). Endocr J 2021;68:129-36.

7. Hubalewska-Dydejczyk A, Duntas L, Gilis-Januszewska A. Pregnancy, thyroid, and the potential use of selenium. Hormones (Athens) 2020;19:47-53.

8. Gan T, Randle RW. The Role of Surgery in Autoimmune Conditions of the Thyroid. Surg Clin North Am 2019;99:633-48.

9. Armangue T, Spatola M, Vlagea A, et al. Frequency, symptoms, risk factors, and outcomes of autoimmune encephalitis after herpes simplex encephalitis: a prospective observational study and retrospective analysis. Lancet Neurol 2018;17:760-72.

10. AlAhmed O, Sivaraman V, Moore-Clingenpeel M, et al. Autoimmune thyroid diseases, autoimmune hepatitis,

Cite this article as: Xiao N, Li Y, Chen W, Liu Y, Li D. Correlation between abnormal thyroid function test and thyroid autoimmunity: a systematic review and meta-analysis. Ann Palliat Med 2021;10(12):12690-12698. doi: 10.21037/apm-21-3534 celiac disease and type 1 diabetes mellitus in pediatric systemic lupus erythematosus: Results from the CARRA Legacy Registry. Lupus 2020;29:1926-36.

11. Jonsdottir B, Larsson C, Carlsson A, et al. Thyroid and Islet Autoantibodies Predict Autoimmune Thyroid Disease at Type 1 Diabetes Diagnosis. J Clin Endocrinol Metab 2017;102:1277-85.

12. Katahira M, Ogata H, Ito $T$, et al. Association of Autoimmune Thyroid Disease with Anti-GAD Antibody ELISA Test Positivity and Risk for Insulin Deficiency in Slowly Progressive Type 1 Diabetes. J Diabetes Res 2018;2018:1847430.

13. Kosmeri C, Siomou E, Challa A, et al. Investigation of Autoimmune Disease in Children with Chronic Spontaneous Urticaria. Int Arch Allergy Immunol 2019;180:250-4.

14. Kyrgios I, Fragou A, Kotanidou EP, et al. DNA methylation analysis within the IL2RA gene promoter in youth with autoimmune thyroid disease. Eur J Clin Invest 2020;50:e13199.

15. Osorio JC, Ni A, Chaft JE, et al. Antibody-mediated thyroid dysfunction during T-cell checkpoint blockade in patients with non-small-cell lung cancer. Ann Oncol 2017;28:583-9.

16. Song J, Shan Z, Mao J, et al. Serum polyamine metabolic profile in autoimmune thyroid disease patients. Clin Endocrinol (Oxf) 2019;90:727-36.

17. Sovetkina A, Nadir R, Scalfari A, et al. Development of Autoimmune Thyroid Disease in Multiple Sclerosis Patients Post-Alemtuzumab Improves Treatment Response. J Clin Endocrinol Metab 2020;105:dgaa453.

18. Zhao F, Feng J, Li J, et al. Alterations of the Gut Microbiota in Hashimoto's Thyroiditis Patients. Thyroid 2018;28:175-86.

19. Tritou I, Vakaki M, Sfakiotaki R, et al. Pediatric thyroid ultrasound: a radiologist's checklist. Pediatr Radiol 2020;50:563-74.

20. Barbuti M, Carvalho AF, Köhler CA, et al. Thyroid autoimmunity in bipolar disorder: A systematic review. J Affect Disord 2017;221:97-106. 